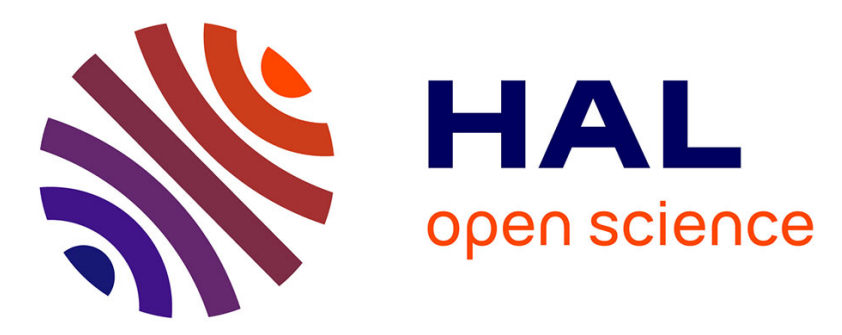

\title{
Un instrument de la politique agraire de Godoy: le Semanario de Agricultura y Artes dirigido a los Párrocos (1797-1808)
}

Elisabel Larriba

\section{- To cite this version:}

Elisabel Larriba. Un instrument de la politique agraire de Godoy: le Semanario de Agricultura y Artes dirigido a los Párrocos (1797-1808). Bulletin Hispanique, 2002, Hommage à François Lopez, 104 (n¹), pp.243-261. 10.3406/hispa.2002.5110 . halshs-01549301

\section{HAL Id: halshs-01549301 https://shs.hal.science/halshs-01549301}

Submitted on 19 Jun 2020

HAL is a multi-disciplinary open access archive for the deposit and dissemination of scientific research documents, whether they are published or not. The documents may come from teaching and research institutions in France or abroad, or from public or private research centers.
L'archive ouverte pluridisciplinaire HAL, est destinée au dépôt et à la diffusion de documents scientifiques de niveau recherche, publiés ou non, émanant des établissements d'enseignement et de recherche français ou étrangers, des laboratoires publics ou privés. 


\section{Un instrument de la politique agraire de Godoy: le Semanario de} Agricultura y Artes dirigido a los Párrocos (1797-1808)

\section{Elisabel Larriba}

\section{Citer ce document / Cite this document :}

Larriba Elisabel. Un instrument de la politique agraire de Godoy: le Semanario de Agricultura y Artes dirigido a los Párrocos (1797-1808). In: Bulletin Hispanique, tome 104, n¹, 2002. pp. 243-261;

doi : $10.3406 /$ hispa.2002.5110

http://www.persee.fr/doc/hispa_0007-4640_2002_num_104_1_5110

Document généré le 15/06/2016 


\begin{abstract}
The Semanario de Agricultura y Artes dirigido a los párrocos (1797-1808), which was a semi-official paper created through Godoy's initiative, was part of an ambitious educational program on economies and politics which was aiming to modernize the farming world. This article will specify what the publishers goals were and what means these public law specialists at the service of the Government, of the public well-being and Enlightment undertook to achieve their aim.
\end{abstract}

\title{
Résumé
}

Le Semanario de Agricultura y Artes dirigido a los Párrocos (1797-1808), périodique semi-officiel créé à l'initiative de Godoy, s'inscrit dans un vaste programme d'éducation économico-politique visant à moderniser le monde agraire. La finalité de cet article est de préciser quels étaient les objectifs des éditeurs et quels furent les moyens que ces publicistes, au service du pouvoir, du bien public et des Lumières, mirent en œuvre pour atteindre leur but.

\section{Resumen}

El Semanario de Agricultura y Artes dirigido a los Párrocos (1797-1808), periódico semi-oficial creado bajo el impulso de Godoy, forma parte de un amplio programa de educación económico-político destinado a modernizar el mundo agrario. Este artículo pretende precisar cuáles eran los objetivos de los editores y de qué medios estos publicistas, al servicio del poder, del bien público y de las Luces, se valieron para concretar sus ideales. 


\title{
Un instrument de la politique agraire de Godoy : le Semanario de Agricultura y Artes dirigido a los Párrocos (1797-1808)
}

\author{
ELISABEL LARRIBA \\ Université de Provence - UMR TELEMME
}

Le Semanario de Agricultura y Artes dirigido a los Párrocos (1797-1808), périodique semi-officiel créé à l'initiative de Godoy, sinscrit dans un vaste programme d'éducation économico-politique visant à moderniser le monde agraire. La finalité de cet article est de préciser quels étaient les objectifs des éditeurs et quels furent les moyens que ces publicistes, au service du pouvoir, du bien public et des Lumieres, mirent en auvre pour atteindre leur but.

El Semanario de Agricultura y Artes dirigido a los Párrocos (1797-1808), periódico semi-oficial creado bajo el impulso de Godoy, forma parte de un amplio programa de educación económico-político destinado a modernizar el mundo agrario. Este articulo pretende precisar cuáles eran los objetivos de los editores y de qué medios estos publicistas, al servicio del poder, del bien público y de las Luces, se valieron para concretar sus ideales.

The Semanario de Agricultura y Artes dirigido a los pàrrocos (1797-1808), which was a semi-official paper created through Godoy's initiative, was part of an ambitious educational program on economics and politics which was aiming to modernize the farming world. This article will specify what the publisher' goals were and what means these public law specialists at the service of the Government, of the public well-being and Enlightment undertook to achieve their aim.

Mots-clés : Presse - Agriculture - Clergé - Lumières - Godoy.

B. $H i ., \mathrm{n}^{\circ} 1$ - juin 2002 - p. 243 à 261. 


\title{
I. - LES OBJECTIFS DU SEMANARIO DE AGRICULTURA Y ARTES...
}

\begin{abstract}
T E SEMANARIO de Agricultura y Artes dirigido a los Párrocos, périodique 1 semi-officiel, créé en 1797, sous l'égide et à l'initiative de Godoy, s'inscrit dans un vaste programme d'éducation économico-politique dont il constitue l'une des pièces maîtresses ${ }^{1}$. A ce titre, il peut sans l'ombre d'un doute être considéré comme l'archétype d'une presse éclairée qui, fortement spécialisée, se veut avant tout utilitaire. Le but des éditeurs et avec eux du Prince de la Paix est de contribuer au développement de l'agriculture et de toute activité économique en relation avec le monde rural. Cet essor apparaît d'ailleurs comme une condition sine qua non à la prospérité du pays, ce qui, d'emblée, est clairement stipulé dans l'Introduction et le Prospectus de l'œuvre. Pour eux, "sin labradores, sin artes y oficios, no sólo no puede florecer el estado, pero ni existir" ${ }^{2}$. Dès lors, il convient de mettre à l'honneur les classes productrices $^{3}$ qui jusqu'alors ont été injustement méprisées. En fait, l'objectif visé est de rétablir un juste équilibre dans une société où les villes font l'objet de toutes les attentions et ce, au détriment du monde rural, ce que souligna Godoy dans la circulaire qu'il adressa en 1796 aux prélats et que les rédacteurs du Semanario... ne manquèrent pas de publier :
\end{abstract}

Siempre ha visto el Rey con sentimiento que la muy apreciable clase de labradores esté abandonada a sus escasos conocimientos en la agricultura y que todos los cuidados, los auxilios y establecimientos benéficos se prodiguen en las ciudades, como si ellas solas fuesen los pueblos privilegiados que mereciesen toda la atención del gobierno, y no debiese ésta recaer más bien sobre las aldeas y pueblos pequeños, en donde suelen faltar medios y luces para mejorar la suerte de sus habitantes. S. M. ama indistintamente a sus vasallos ${ }^{4}$.

Dans cette perspective, et à l'invite du pouvoir, les rédacteurs du périodique se fixèrent trois objectifs principaux :

1. Sur le Semanario de Agricultura y Artes... on pourra consulter Fernando Díez Rodríguez, Prensa agraria en la España de la ilustración : El Semanario de Agricultura y Artes dirigido a los Párrocos (1797-1808), Madrid, Ministerio de Agricultura, 1980 et l'introduction de l'anthologie que nous avons réalisée en collaboration avec Gérard Dufour : El Semanario de Agricultura y Artes dirigido a los Párrocos (1797-1808). Antologia, Valladolid, Editorial Ambito, 1997.

2. "Introducción» in Sernanario de Agricultura y Artes..., Madrid, tome I, 1797, p. III (ou p. 69 de notre anthologie).

3. Id.

4. Lettre du Prince de la Paix aux évêques, San Lorenzo, 28 novembre 1796, in Semanario de Agricultura y Artes..., tome I, p. IV-V (ou p. 70-71 de notre anthologie). 
- le premier était d'informer les paysans des nouvelles techniques agraires utilisées tant en Espagne qu'à l'étranger et de permettre ainsi l'amélioration des méthodes alors en vigueur. D'où la publication de bon nombre de textes empruntés à des auteurs étrangers ou rendant compte de leurs travaux ;

- le deuxième consistait à lutter contre le poids écrasant de la tradition, des pratiques ancestrales, des croyances stupides qui reposent sur un lourd substrat de superstition et sont inéluctablement une entrave au progrès de la nation ;

- le troisième, enfin, était de favoriser la communication entre les diverses régions d'Espagne en leur offrant un organe d'expression commun et de portée nationale. Le journal, tel qu'il avait été conçu, se devait d'être un lieu d'échanges, un espace d'expression ouvert à tout un chacun. A ce titre, il apparaît comme une ouvre collective où la participation du public, vivement sollicitée, est perçue comme une priorité absolue ${ }^{5}$.

\section{II. - LES MOYENS MIS EN CEUVRE}

\section{Se mettre à la portée du public}

Compte tenu de ses objectifs, le journal, qui s'adressait à un public atypique, le monde rural, se devait impérativement d'être divulgatcur ct didactique. De ce fait, les rédacteurs insisteront haut et fort sur la nécessité de concevoir les articles dans "un lenguaje inteligible a los sencillos aldeanos" ${ }^{6}$. C'est dans cette optique que sera publié, par exemple, dans le numéro 6 du Semanario..., en date du 9 février 1797, un écrit que l'on doit à l'Evêque de Plasencia (par ailleurs directeur de la Société Economique de Trujillo) et dont le titre est des plus significatifs : Cartilla rústica sobre las utilidades del sacho y la escarda en las labores de Extremadura, dispuesta en preguntas y respuestas por la real Sociedad económica de Trujillo, y acomodada al estilo y capacidad de los

5. Sur ce sujet on pourra consulter : Elisabel Larriba, "Contribution du clergé à la rédaction du Semanario de Agricultura y Artes dirigido a los Párrocos, in L'Espagne du $X V I I I^{e}$ siècle. Economie, sociéte, idéologie, culture. Actes des journées d'étude sur "Ville et campagne» et sur les Cartas marruecas des 5 et 6 décembre 1997. Sous la direction de Jacques Soubeyroux, Publications de l'Université de Saint-Ftienne, 1997, p. 217-233 et Id., "Un intento de reforma agraria por y para las clases productoras: el Semanario de Agricultura $y$ Artes dirigido a los Párrocos (1797-1808)", in Brocar. Cuadernos de investigación historica, Universidad de la Rioja, $\mathrm{n}^{\circ} 23$ (1999), p. 87-117.

6. Archivo Histórico Nacional, Estado, legajo 3485 : Lettre de Juan Bautista Virio (l'un des éditeurs du journal) au Prince de la Paix, Madrid, 22 janvier 1797. 
labradores de esta ciudad y su partido ${ }^{7}$. On retrouve d'ailleurs ce même souci de se mettre à la portée d'un public, ô combien particulier, chez d'autres ilustrados soucieux de contribuer au développement du monde agraire, parmi lesquels Jovellanos qui, dans son Informe sobre la Ley agraria, prônait la création de "cartillas técnicas, en estilo llano y acomodado a la comprensión de un labriego» 8 et souhaitait que les curés devinssent «los padres e institutores de sus pueblos» ${ }^{9}$. Toutefois, entre les déclarations d'intention et la réalité, se creuse bien souvent un abîme. Et force est de constater que les rédacteurs ne surent pas toujours respecter cette ligne directrice qu'ils s'étaient fixée lors de la création du journal. Ainsi, lorsqu'en 1805 le périodique passe sous la direction du Real Jardin Botánico de Madrid, il tend à oublier ses visées divulgatrices pour se transformer progressivement en revue scientifique faite par et pour des spécialistes ${ }^{10}$.

\section{Prêcher par l'exemple}

Les éditeurs, s'ils veulent que leur entreprise soit couronnée de succès, se doivent de trouver le ton juste pour s'adresser à un public hors normes, essentiellement rural, et pour être à même de le convaincre de la pertinence des enseignements, des techniques véhiculées par le périodique. En fait, il s'agit pour eux, ce qui ne sera pas chose facile, de venir à bout des réticences d'une frange de la population qui, généralement, se caractérise par sa méfiance, si ce n'est son hostilité à toute forme d'innovation. Il s'agit de lutter contre le poids d'une tradition ancestrale face à laquelle les mots ne peuvent suffire. Il ne s'agit pas de dire, il faut également démontrer et démontrer par les faits. La théorie est évidemment indispensable. Toutefois, pour qu'elle puisse porter ses fruits, elle doit obligatoirement aller de pair avec l'expérience : sans l'exemple, point de salut. Les rédacteurs du Semanario... en sont parfaitement conscients et ils ne manquèrent pas de le souligner dans leur premier article : Del estudio de la agricultura, qu'ils

7. Semanario de Agricultura y Artes dirigido a los Párrocos, tome I, n 6, 9 février 1797, p. 81-93.

8. Gaspar Melchor de Jovellanos, Informe sobre la Ley agraria, Ed. de Guillermo Carnero, Madrid, Cátedra, 1997, p. 403 et sq.

9. Id., p. 404.

10. Elisabel Larriba, Gérard Dufour, introduction à l'anthologie du Semanario, op. cit., p. 25-36. 
empruntent à la version espagnole du Dictionnaire de l'abbé Rozier ${ }^{11}$, et où l'on peut lire : «A este primer estudio [l'étude de l'Agriculture] debe suceder el de la aplicación de las doctrinas a la experiencia, sin la cual la más excelente teoría, no es más que charlatanería sin fundamento, que contradice y destruye la menor variación en las circunstancias locales» ${ }^{12}$. Et les rédacteurs de poursuivre : «Predíquese con ejemplos, y no con palabras, y se conseguirá la más sólida y única instrucción que se puede dar a los labradores, que no leen, ni tal vez saben, pero observan el buen o mal éxito de las experiencias que otros hacen, y éste es el único libro que leen y comprenden bien» ${ }^{13}$.

Mettant ces principes en application, les rédacteurs du Semanario... vont tenter, dans la mesure du possible, de multiplier les écrits où sont présentées les expériences réalisées par tel ou tel individu, par telle ou telle institution. Pour ne citer qu'un exemple, dans le numéro 333, en date du 19 avril 1803, figure un article où l'on trouve le descriptif détaillé d'une batteuse, planche à l'appui, ce qui, par ailleurs, est tout à fait exceptionnel pour la presse espagnole de l'époque ${ }^{14}$. Eh bien, ce premier article purement technique va donner lieu, quelques semaines plus tard (dans le $\mathrm{n}^{\circ} 348$ ), à la publication d'un second intitulé cette fois-ci : Utilidad del volvedor de mieses publicado en el núm. $333^{15}$. Le sujet est donc repris mais sous un angle différent. En fait, il est désormais question de démontrer de manière irréfutable que cette nouvelle machine constitue un réel progrès et peut, par voie de conséquence, être utile. D'où la publication d'une lettre où le curé de Tudelilla (villa de 618 habitants, dans la province de Burgos ${ }^{16}$ ) qui, de sa propre initiative et

11. Curso completo o Diccionario universal de Agricultura teórica, práctica, Económica, y de medicina rural y veterinaria. Escrito en francés por una Sociedad de Agronomos, y ordenado por el Abate Rozier. Traducido al castellano por Don Juan Alvarez Guerra, individuo en la clase de Agricultura de la Real Sociedad Económica de Madrid. Con superior permiso, Madrid, Imprenta Real, por D. Pedro Julián Pereyra, Impresor de Cámara de S.M., 1797-1803.

12. "Del estudio de la Agricultura", in Semanario de Agricultura y Artes dirigido a los Párrocos, tome I, $\mathrm{n}^{\circ} 1,5$ janvier 1797, p. 5.

13. Ibid., p. 5-6.

14. "Descripción de un nuevo volvedor de mieses para trillar", in Semanario de Agricultura y Artes dirigido a los Párrocos, tome XIII, n 333, 19 mai 1803, p. 305-308.

15. «Utilidad del volvedor de mieses publicado en el núm. 333 - Extracto de una carta de DPJH, cura párroco de Tudelilla, en el obispado de Calahorra", in Semanario de Agricultura $y$ Artes dirigido a los Párrocos, tome XIV, $\mathrm{n}^{\circ} 348,1$ septembre 1803 , p. 143-144.

16. Censo de Floridablanca, 1787. 
sur ses fonds personnels, fit construire et tester la machine en présence de tous les paysans de sa paroisse, rend compte des excellents résultats obtenus et de l'impact produit sur les témoins de l'expérience :

Esto les hace mirar ya sin repugnancia las instrucciones del semanario, porque no hay móbil más eficaz que el interés. Por eso quisiera yo que con los sujetos de algún caudal, de que tan prodigamente suelen abusar, dedicasen alguna parte a la ejecución de los experimentos que se nos proponen y así se convencerían fácilmente de sus errores hasta los más idiotas, y abrazarían los medios de adelantar su prosperidad particular y la del estado, como se ve ahora en este pueblo en que todos quieren tener la máquina cuyas ventajas han apreciado. Extraño mucho que los curas se hagan tan sordos a las benéficas intenciones del Soberano comunicadas por los señores Obispos, pues en más de 100 pueblos de la Rioja alta y baja, y parte de Navarra no se sabe que haya tal periódico en el mundo, y era menester obligar a los curas a que lo tomasen (pues nada les cuesta, sino a sus iglesias) para que valiéndose de sus lecciones, procurasen adelantar la agricultura y la industria, y se abstuviesen de censurar los buenos deseos de los pocos que se toman el trabajo de hacer valer las verdades experimentadas ${ }^{17}$.

Nous nous trouvons là dans une logique typiquement éclairée, puisque placée sous le sceau de l'expérience, des vérités prouvées, de l'empirisme et donc de la raison, par opposition au poids des autorités, des préjugés (des preocupaciones - pour employer la terminologie de l'époque), du dogmatisme et de l'irrationalité. Tout aussi significatifs sont les commentaires faits par les éditeurs dans le numéro 15, publié le 13 avril 1797, alors qu'ils venaient de recevoir un courrier émanant d'un ecclésiastique «muy respetable» les priant de recommander l'usage d'une mixture à base de lierre grimpant aux miraculeuses vertus médicinales. Faisant part aux lecteurs de l'information reçue, les responsables de la publication s'empressèrent de rajouter :

Aunque estamos muy persuadidos de la recta intención y buenos deseos de quien nos comunica esta receta, nos guardaremos bien de recomendarla, mientras la experiencia, y una análisis bien hecha de dichos polvos, no nos den más luces; y aun entonces debe la prudencia modificar a las circunstancias el uso de las medicinas; que eso de que tales polvos o tales píldoras curan todas o algunas enfermedades, sin distinción de sexo, edad o temperamento, es para que lo crean Hotentotes, no gentes civilizadas. El mismo sabio eclesiástico que recomienda estos polvos, advierte que se pudieran hacer algunas pruebas para asegurarse de su virtud ${ }^{18}$.

17. «Utilidad del volvedor de mieses publicado en el núm. 333 - Extracto de una carta de DPJH, cura párroco de Tudelilla, en el obispado de Calahorra», op. cit., p. 143.

18. Semanario de Agricultura y Artes dirigido a los Párrocos, tome I, $\mathrm{n}^{\circ} 15,13$ avril 1797 , p. 234. 
Force est de constater combien les rédacteurs ont à cœur de porter un regard critique sur l'information reçue, combien ils accordent de l'importance à l'expérience. Avant de se prononcer, ils exigent non seulement l'obtention d'une preuve mais également d'une contre-preuve et en cela ils adoptent une démarche qui se veut rigoureuse et scientifique. Cette volonté affirmée de combattre les erreurs par des vérités prouvées, cette foi, cet optimisme en la raison comme moyen d'accéder au bonheur apparaît, notamment, dans un article publié en 1803 sous le titre de Reflexiones sobre uno de los artículos de B. Franklin ${ }^{19}$ et dont l'auteur, un certain Roberto de Armesto y Silva, officier de la marine, se présentant en qualité de «apasionado y constante suscriptor» du journal livre diverses considérations sur la vie et la mort. Suite à l'épidémie de peste qui avait frappé Cadix, celui-ci avait décidé de procéder à diverses expériences sur des mouches afin de trouver un palliatif à ce mal. Faisant part de ses résultats, il déclarait :

Todo el arte consistiría entonces en conocer de qué especie era el enemigo, para oponerle su contrario. Pero es menester hacer experiencias para sentar principios que hoy se suponen demasiado gratuitamente. Por ejemplo, se sienta que el veneno de la peste es un alcalí y que en las fiebres putridas convienen los acidos... ¿Pero cómo se sabe lo primero? 20

Et, alors que Franklin aurait désiré ressusciter un siècle plus tard, grâce aux progrès de la science, Roberto de Armesto y Silva, si la possibilité lui en était donnée, souhaiterait pouvoir vivre cent ou deux cents ans de plus afin de pouvoir apprécier les bienfaits des Lumières :

Bien me hago cargo - disait-il - de que ya no hay Arístides ni Catones que seguir; pero percibo la aurora de un bello día para nuestra patria. Sí, la luz empieza a esparcirse, y sus rayos van a combatir las sombras fugitivas de la ignorancia, hasta en los senos más retirados y escondidos. Quisiera yo ir viendo a la claridad de este día que amanece las preocupaciones destruidas, proscritas las bárbaras instituciones, las útiles y benéficas propagadas; quisiera ver cómo la agricultura en honor rodeada de las ciencias y las artes, iba preparando por dos o tres generaciones el grado de esplendor y prosperidad a que nuestra nación está llamada por la naturaleza, desde que la destinó este suelo y este clima, y la dotó con el carácter de sus individuos. ¡Esto quisiera yo ver, señores editores, y tener en ello siquiera tanta parte como Vms.! ;Dios les guarde, si no puede ser doscientos ni cien años, cuando menos lo bastante para dejar tan adelantada su empresa que no sea posible a los enemigos del bien volverla atrás! Esto lo espera ver también su apasionado y constante suscriptor ${ }^{21}$.

19. "Reflexiones sobre uno de los artículos de B. Franklin", in Semanario de Agricultura y Artes dirigido a los Párrocos, tome XIV, ${ }^{\circ}$ 363, 15 décembre 1803, p. 381-383.

20. Ibid., p. 382.

21. Ibid., p. 383. 
Il ne fait aucun doute que l'optimisme de ce souscripteur est aussi celui des rédacteurs du Semanario de Agricultura y Artes... qui, pour le bien de la nation et au nom de la sacro-sainte utilité, souhaitaient s'ériger en correcteurs des erreurs que pouvaient commettre leurs compatriotes. C'est ainsi, et là encore il ne s'agit que d'un exemple parmi tant d'autres, qu'à une époque où certains ecclésiastiques proclament que ceux qui pratiquent l'inoculation ne sont pas dignes de l'absolution, le journal, partant en campagne contre l'ignorance, publiera divers articles rendant compte des progrès de la médecine en matière de vaccination en s'appuyant sur des exemples concrets. Car on ne peut admettre, comme le souligne un souscripteur, que mille enfants soient morts de la variole dans la seule ville de Ségovie, tout simplement parce que leurs parents, abusés par de fausses autorités, se sont refusés à les faire vacciner ${ }^{22}$.

\section{Privilégier l'observation qui permet d'accéder à la connaissance}

Pour combattre cette ignorance endémique qui paralyse le développement de l'Espagne, il ne suffit pas de se plonger dans la lecture de savants traités sur l'agriculture, les arts ou bien encore le commerce et d'en rendre compte. La diffusion du savoir doit être précédée par un long et indispensable travail d'observation sur le terrain. Les rédacteurs du Semanario de Agricultura y Artes... en sont persuadés et l'on retrouve là, une fois de plus, l'une des caractéristiques des Lumières : l'importance accordée à l'observation et par voie de conséquence aux voyages didactiques, aux voyages d'études. En fait, si l'on veut être à même d'offrir un remède efficace, il faut avant tout connaître avec précision la nature exacte des maux que l'on combat. Il convient donc, au préalable, de s'offrir les moyens d'apprécier à sa juste valeur l'état des différentes activités économiques du pays, de son agriculture, de sa situation démographique, de ses centres d'enseignement... Avant tout, il faut pallier un cruel manque d'informations et mettre un terme à une situation où l'on ignore ce qui se passe non pas à l'étranger mais tout simplement dans la province voisine. Dès lors, le Semanario de Agricultura y Artes dirigido a los Párrocos se doit d'établir la communication non seulement entre les différentes régions d'Espagne, mais également entre la ville et la campagne dont le sort est intimement lié, point sur lequel les

22. "Carta de un suscriptor de Segovia sobre viruelas", in Semanario de Agricultura y Artes dirigido a los Párrocos, tome IV, $\mathbf{n}^{\circ}$ 97, 8 novembre 1798, p. 286. 
UN INSTRUMENT DE LA POLITIQUE AGRAIRE DE GODOY

éditeurs avaient insisté d'emblée dans le prospectus de l'œuvre où ils déclaraient :

Subsistirá por desgracia la división que introdujo entre los hombres la formación de las ciudades. Subsistirán éstas a pesar de sus vicios. Pero entre tanto estudio frívolo como nos entretiene en ellas, entre tantas ocupaciones estériles como nos hacen perder el tiempo, ¿será posible que (sordos siempre a las voces de la gratitud) no haya entre nosotros algunos que, aprovechándose de la concurrencia de luces que se halla sólo en los pueblos grandes, no traten de corresponder a los indecibles beneficios que debemos a la clase que nos sustenta, comunicándoles estas mismas luces, haciéndola apreciar su dignidad e importancia, instruyéndola en los descubrimientos que la puedan ser útiles, y finalmente en cuanto haya de mejorar su suerte? Tal sería el medio de que los campos se utilizasen de las grandes poblaciones, haciendo éstas refluir en ellos los adelantamientos de las ciencias que sólo se pueden perfeccionar en las ciudades populosas, y tal el fin que se debe proponer un sabio gobierno para que los miembros del estado se auxilien mutuamente como los de cuerpo ${ }^{23}$.

Autrement dit, il est ici question de rejeter une vision dichotomique où ville et campagne s'opposent au profit d'un système de pensée reposant sur les notions d'unité et d'interdépendance. Et l'on ne pourra y parvenir que grâce à une meilleure connaissance de la réalité espagnole, ce que ne manquèrent pas de souligner les rédacteurs :

Casi todas las naciones de Europa han abierto los ojos en esta parte. Parece que los hombres desengañados ya de los desvaríos en que han divagado tanto tiempo en materia de estudios, vuelven como de un penoso viaje a reconocer su casa, la economía de ella, sus tierras, sus frutos, el modo de aumentarlos y en suma cuanto les rodea e interesa a su bienestar ${ }^{24}$.

Et c'est dans cette perspective que les rédacteurs, en quête d'informations permettant de dresser un bilan de santé des différentes régions d'Espagne, vont élaborer, à l'intention des lecteurs du journal, un questionnaire détaillé regroupant 47 points, qui sera inséré dans le numéro 92 du Semanario... ${ }^{25}$. Par ailleurs, soucieux de créer un climat d'émulation, ils n'hésiteront pas à ériger en exemple des hommes ayant pris la peine d'observer leur environnement. C'est ainsi que dans le numéro 21, en date du 25 de mai 1797, est

23. "Prospecto", in Semanario de Agricultura y Artes dirigido a los Párrocos, tome I, 1797, p. VIII-IX (ou p. 74 de notre anthologie, op. cit.)

24. Ibid., p. IX (ou p. 74 de notre anthologie).

25. "Medios para conocer el estado de nuestra agricultura» in Semanario de Agricultura y Artes dirigido a los Párrocos, tome IV, $\mathrm{n}^{\circ}$ 92, 4 novembre 1798. Ce document est en partie reproduit in Fernando Díez Rodríguez, op. cit., p. 190-191. 
publié l'Extracto de una carta a los Editores que l'on doit à un certain Francisco Hernández González, bénéficier d'Alba de Tormes, et dans lequel on peut lire en introduction :

Don Francisco Hernández González, Beneficiado de Alba de Tormes, escribe a los Editores del Semanario, que ha hecho viajes a varios pueblos de España para enterarse y aprender cuanto se ha adelantado en diversos ramos de agricultura y de industria, llevando intención de ponerlos después en práctica, y de enseñar a sus consúbditos, como es tan propio de su estado; y añade que la persuasión de que las ocupaciones útiles premian por sí ampliamente a los hombres activos, pues les proporcionan mucha variedad de goces y convenencias, y disipan la miseria, que unida a la ociosidad es el origen de los vicios, le hizo perseverar en su loable empeño con la mayor constancia ${ }^{26}$.

On retrouve donc ici la défense d'un processus en deux temps fondé, d'une part, sur une recherche de la connaissance qui n'est en aucun cas livresque mais pratique puisqu'elle repose sur l'observation, et, d'autre part, sur une transmission de la connaissance tout aussi indissociable de la pratique.

Compte tenu de la ligne de conduite fixée par les auteurs du Semanario de Agricultura y Artes..., on ne s'étonnera pas d'y trouver plusieurs articles rendant compte de voyages agronomiques, ce qui s'inscrit parfaitement dans le droit fil de la tradition éclairée. Articles la plupart du temps intulés Observaciones sobre... Ainsi, le numéro 472, du 16 janvier 1806, s'ouvre sur Las Observaciones de Agricultura hechas desde Ocaña hasta Huete en Julio, Agosto y Septiembre de $1803^{27}$, texte signé par Esteban Boutelou qui avait à cœur de rappeler que «uno de los grandes medios que emplea la política para promover e ilustrar la Agricultura, es el de los viajes agronómicos» ${ }^{28}$. Ces voyages, souligne-t-il, doivent permettre de «reconocer el estado de la Agricultura en diversas provincias, los cultivos que en ésta o en aquélla deben promoverse, los que conviene abandonar $y$ las prácticas que es

26. "Extracto de una carta a los Editores", in Semanario de Agricultura y Artes dirigido a los Párrocos, tome $\mathrm{I}, \mathrm{n}^{\circ} 21,25$ mai 1797, p. 330-332.

27. «Las Observaciones de Agricultura hechas desde Ocaña hasta Huete, en Julio, Agosto y Septiembre de 1803", in Semanario de Agricultura y Artes dirigido a los Párrocos, tome 19, $\mathrm{n}^{\circ} 472,16$ janvier 1806 , p. 33- 41. Larticle se poursuit dans les numéros 474 (30-1-1806, p. 77-80), 475 (6-2-1806, p. 89-92), 476 (13-2-1806, p. 103-109) et 477 (20-2-1806, p. 119-124).

28. Ibid., $\mathrm{n}^{\circ} 472,16$ janvier 1806, p. 33. 
necesario reformar, o ventajoso hacerlas generales" ${ }^{29}$. C'est ainsi qu'il s'est attaché à "hacer observaciones sobre la naturaleza del terreno y su aptitud para estas o las otras producciones, y recoger noticias interesantes para la Agricultura, prescindiendo por ahora de varias indagaciones botánicas, y del hallazgo que se me proporcionó, de muchas plantas preciosas» ${ }^{30}$. Et c'est le fruit de ses observations qu'il expose dans le Semanario... Dans le même ordre d'idées, il publia en 1807 Varias observaciones sobre la Agricultura de Jadraque ${ }^{31}$ puis, l'année suivante, les Observaciones sobre las grandes labores de Andalucia, e ideas sobre las utilidades y perjuicios que resultan al Estado y a la Agricultura de la excesiva extensión de los grandes cortijos ${ }^{32}$. Toutefois, si la connaissance de l'Espagne s'avérait indispensable, les rédacteurs ne négligèrent pas pour autant de porter un regard curieux sur l'étranger, et selon le même principe, ils publièrent en 1807 un long article intitulé Observaciones hechas en un viaje veterinario al imperio de Marruecos ${ }^{33}$ et ce, à l'invite du Prince de la Paix ${ }^{34}$. De même, l'importance capitale qu'ils accordaient à l'observation et aux voyages agronomiques, pratique qu'ils espéraient voir développer en Espagne, les amena à insérer dans les pages de leur journal un Extracto de lo más notable de los viajes de Arthur Young en Inglaterra qui couvrait pas moins de sept numéros consécutifs ${ }^{35}$. L'œuvre de l'Anglais, admirable et régie par des principes dignes d'être suivis, méritait, selon eux, d'être connue par les Espagnols, en dépit des différences majeures qui oppo-

29. Ibid., p. 35.

30. Ibid., p. 33.

31. "Varias observaciones sobre la Agricultura de Jadraque», in Semanario de Agricultura y Artes dirigido a los Párrocos, tome XIX, ${ }^{\circ}$ 536, 9 avril 1807, p. 217-224.

32. "Observaciones sobre las grandes labores de Andalucía, e ideas sobre las utilidades y perjuicios que resultan al Estado y a la Agricultura de la excesiva extensión de los grandes cortijos", in Semanario de Agricultura y Artes dirigido a los Párrocos, tome XXIII, n 590, 21 avril 1808, p. 241-252.

33. "Observaciones hechas en un viaje veterinario al imperio de Marruecos", in Semanario de Agricultura y Artes dirigido a los Párocos, tome XXII, n 549, 9 juillet 1807, p. 17-22; $\mathrm{n}^{\circ} 550,16$ juillet 1807, p. $45-48$ et $\mathrm{n}^{\circ} 551,23$ juillet 1807, p. 54-59.

34. Ibid., p. 17 : «Publícanse de orden del Serenísimo Señor Príncipe Generalísimo Almirante, que no contento con haber introducido en nuestra Agricultura muchas de las producciones más preciosas del Africa, aspira a mejorar las razas de todo género de ganados, y a propagar las que nos faltan."

35. "Extracto de lo más notable de los viajes de Arthur Young en Inglaterra», in Semanario de Agricultura y Artes dirigido a los Párrocos, tome IX, $\mathrm{n}^{\circ}$ 217, 26 février 1801, p. 121-134; $\mathrm{n}^{\circ} 218,5$ mars 1801 , p. $137-250 ; \mathrm{n}^{\circ} 219,12 \operatorname{mars} 1801$, p. 153-165; $\mathrm{n}^{\circ} 220,19$ mars 1801 , p. 169-182; $\mathrm{n}^{\circ} 221,26$ mars 1801 , p. 185-198; $\mathrm{n}^{\circ} 222,2$ avril 1801 , p. 201-214 et $\mathrm{n}^{\circ} 223,9$ avril 1801, p. $217-223$. 
saient l'agriculture des deux pays. Ainsi, après avoir précisé en note au titre de l'article : «Este viaje puede servir de modelo para los que entre nosotros quieran emprender otros semejantes» ${ }^{36}$, procédèrent-ils, en introduction, à un portrait fort élogieux de Young tout en mettant l'accent sur les méthodes qui étaient les siennes :

Young es tal vez el labrador más sabio que hoy tiene Europa; y aunque la agricultura inglesa sea en gran parte inaplicable a nuestro clima, no será desagradable a nuestros cultivadores el tener alguna idea de las obras de este célebre escritor, que continúa publicando en su patria los Anales de agricultura. "Yo, dice, fui el primero que hice en Inglaterra un viaje con el fin de dar al público un plan de la agricultura de mi nación, de la fuente de riqueza verdadera, y de la reforma del sistema político cubierto de orín gótico, de preocupación y errores. El conocimiento práctico de cuanto se ejecuta en todo el reino facilitará la decisión de muchos puntos importantes sobre los cuales sólo se pueden formar conjeturas; y no sabiendo los males, no es posible que se aplique el remedio. En un distrito se enriquecen los labradores mediante algunas prácticas que enriquecerían a los de otros que las ignoran; y mi intento es generalizar estos conocimientos locales, aunque temo que de mil agricultores apenas habrá cinco que me leerán. No se busque en mis escritos correción de estilo, porque escribo en los mesones y chozas de los labradores, y sólo atiendo a la exactitud de mis relaciones... ${ }^{37}$.

\section{La participation du public : une marque de patriotisme}

Cette recherche constante de l'information et cette volonté de toucher du doigt la réalité du terrain, passent également par de constants appels à participation auprès d'un public qui n'est pas perçu comme un simple récepteur mais comme un interlocuteur à part entière. D'où le pari de faire du journal une structure ouverte, une œuvre «interactive» où tout un chacun, quelle que soit sa condition, se doit de participer au nom du bien public. Et c'est là que le concept de patriotisme entre en jeu, concept auquel les rédacteurs feront référence en de multiples occasions. C'est ainsi que dans un Aviso de los redactores inséré dans l'un des tout premiers numéros ${ }^{38}$, les éditeurs rendirent hommage, publiquement, à tous ceux qui, dès le début, avaient apporté leur soutien et leur collaboration au périodique : "Apenas déclaraient-ils - ha comenzado a circular el Semanario de agricultura y artes

36. Ibid., $\mathrm{n}^{\circ} 217,26$ février 1801, p. 121.

37. Id.

38. "Aviso de los redactores», in Semanario de Agricultura y Artes dirigido a los Párrocos, tome I, $\mathrm{n}^{\circ} 12$, p. $190-192$. 
en las provincias del reino, han manifestado varios señores prelados, párrocos, hacendados y labradores su anhelo de contribuir con sus luces al bien de la causa pública, y esparcir noticias útiles particularmente a los apreciables labradores» ${ }^{39}$. En guise de remerciement, les rédacteurs n'hésitèrent pas à publier la liste de ces lecteurs qui ne s'étaient nullement contentés de porter un regard passif sur le journal. En effet, et selon leurs propres termes : "Los editores del Semanario faltarían al agradecimiento debido al patriotismo de estos individuos si dejasen de comunicar al público para la estimación general los nombres de los que se han servido dirigirles sus reflexiones o noticias oportunas" "40. A l'instar de la publication des listes de souscripteurs, pratique fort répandue à l'époque ${ }^{41}$, cette mesure à l'impact publicitaire assuré servait tout autant les intérêts du journal que ceux de ses correspondants dont l'orgueil se trouvait flatté. Les propos tenus par Godoy dans ses Mémoires alors qu'il évoque les motivations de ceux qui se sont enrôlés dans l'armée pour prendre part à la Guerre de la Convention, sont en la circonstance fort significatifs : "un gran número de nuestros nuevos soldados affirmait-il - se han alistado voluntariamente por resultas de los sermones que han oído, o por la vanidad de leer sus nombres en la Gaceta» ${ }^{42}$. Cette assertion est totalement erronée, car les listes de volontaires ne furent jamais publiées dans ce journal ${ }^{43}$. Toutefois, qu'une telle explication ait seulement pu être envisagée nous en dit long sur l'importance accordée au simple fait de voir son nom figurer dans un périodique. Fort logiquement, les rédacteurs du Semanario de Agricultura y Artes..., qui en étaient parfaitement conscients, surent en tirer parti. D'où cet hommage qui figure dans l'un des premiers numéros du journal où la liste nominative à laquelle nous venons de faire référence est accompagnée de la lettre d'un certain Pedro Antonio Fernández, Rector de Santiago de Albarracín. Ce dernier y rapportait comment certains de ses paroissiens s'étaient lancés dans la fabrication de savon, selon la méthode exposée dans l'un des articles du Semanario... et comment ils pensaient pouvoir y apporter quelques améliorations. Aussi, s'engageaitil, si leurs expériences étaient couronnées de succès, à en informer derechef

39. Ibid., p. 191.

40. Ibid., p. 190.

41. A ce sujet voir : Elisabel Larriba, Le Public de la presse en Espagne à la fin du XVIII siecle (1781-1808), Paris, Ed. Champion, 1998.

42. Príncipe de la Paz, Memorias, BAE, Madrid, Real Academia de la Historia, 1965, tome LXXXVIII, p. 86 (a). [ $1^{\text {re }}$ édition en français, Paris, 1836.]

43. Ce point a déjà été noté par C. Seco Serrano dans son édition des Memorias del Principe de la Paz, BAE, tome LXXXVIII, p. 86 (a). 
le journal. S'ensuivait, en guise de conclusion, une note succincte libellée en ces termes:

Si hay muchos que sigan el ejemplo de este celoso eclesiástico en comunicarnos los adelantamientos e industrias de su país, se llenarán completamente las esperanzas del gobierno, y será el Semanario un medio por el que unos a otros nos comunicaremos nuestros adelantamientos en beneficio de la causa pública y de cada uno en particular ${ }^{44}$.

Et l'on retrouve dans cette simple note, qui clôt l'article et le numéro, deux idées fortes chères aux éditeurs : d'une part la volonté de présenter le journal comme un lieu d'échange et, d'autre part, celle de rappeler qu'intérêt général et intérêt particulier vont de pair.

Souhaitant donner la parole au public, les rédacteurs publièrent quelque 252 lettres ce qui représente environ $10 \%$ de la production totale. Ainsi furent-ils 186 individus à s'adresser directement aux éditeurs et par leur entremise aux lecteurs du journal soit en tant que demandeurs d'informations, soit pour faire part de leurs observations de manière spontanée ou en réponse aux appels à participation lancés en de multiples occasions par les responsables de la publication, comme ce fut par exemple le cas en novembre 1806 où l'on pouvait lire dans le numéro 515 la note suivante :

Deseando ilustrar cuanto nos sea posible nuestra Agricultura, y careciendo de datos para ello, suplicamos a los hombres ilustrados y amantes del bien de su país nos comuniquen todo género de noticias relativas a las producciones que en él se cultivan, al método e instrumentos con que se hace, al producto que dan, a las cargas que tienen; en suma sobre todo lo que se practica en los campos, sin omitir la menor circunstancia por despreciable que parezca. Nosotros haremos buen uso de las noticias que se nos dieren, y manifestaremos nuestro reconocimiento a los celosos patricios que se sirviesen comunicárnosla 45 .

Ainsi, grâce à la publication de la correspondance reçue par les éditeurs, c'est bien souvent un jeu de questions et de réponses qui s'établit entre les différentes parties. Réel ou fictif ? Il est parfois bien difficile de trancher car les rédacteurs n'hésitèrent pas, en certaines occasions, à publier de fausses lettres, pratique d'ailleurs courante à l'époque. Il n'en reste pas moins qu'ils

44. "Aviso de los redactores", in Semanario de Agricultura y Artes dirigido a los Párrocos, tome I, n' 12, p. 192.

45. "Aviso", in Semanario de agricultura y Artes dirigido a los Párrocos, tome XX, $n^{\circ} 515$, 13 novembre 1806, p. 314. 
eurent à cœur de mettre sur un plan d'égalité (ou du moins d'en donner l'illusion) leurs informations, leurs articles et ceux d'un public hétéroclite où étaient représentés tous les états, les deux sexes (même si les femmes demeurent minoritaires) et la plupart des classes sociales (de l'évêque au curé de village, du militaire à l'artisan en passant par les laboureurs, les employés de l'État, les médecins, les vétérinaires...). De ce point de vue l'ambition des rédacteurs du Semanario de Agricultura y Artes... de faire un ouvrage pour tous, voire par tous était amplement comblée ${ }^{46}$.

\section{La participation du clergé paroissial}

Enfin le dernier impératif pour les rédacteurs, et non le moindre, était de s'assurer de la collaboration du clergé, et en particulier du clergé paroissial, qui apparaissait comme le seul intermédiaire possible entre le savoir véhiculé par le périodique et le monde rural qui en était le premier destinataire. L'idée d'associer les ecclésiastiques, et notamment ceux qui exerçaient en milieu campagnard, à la diffusion d'un enseignement agraire n'est pas une nouveauté. Depuis longtemps en France on encourageait ces représentants de l'Eglise à devenir des instituteurs agricoles des populations. Henri Louis Duhamel de Monceau (membre de l'Académie des Sciences de Paris), qui s'intéressait tout particulièrement à l'instruction des paysans ${ }^{47}$ déclarait ainsi dans les Elements d'Agriculture:

Il n'est point de curé dans les campagnes, qui après avoir rempli les devoirs de son état n'ait de moments de loisirs : plusieurs d'entre eux pensent que le meilleur emploi qu'ils en puissent faire est d'instruire leurs paroissiens des pratiques utiles qu'ils ont trouvées détaillées dans les bons ouvrages d'agriculture, en faisant le choix de ce qui peut être applicable à la nature de leur terrain et en employant dans des conversations fructueuses un langage approprié à la manière de concevoir des habitants des campagnes, pour rendre plus sensibles les objets qu'ils voudront leur inculquer et qui, sans cet officieux secours, se trouveraient au-dessus de la portée de leur esprit ${ }^{48}$.

46. Sur ce point consulter notre article: "Un intento de reforma agraria por y para las clases productoras: el Semanario de Agricultura y Artes dirigido a los Párrocos (1797-1808)", Brocar. Cuadernos de investigación histórica, op. cit.

47. Henry Louis Duhamel de Monceau publia notamment un ouvrage intitulé École d'Agriculture, Paris, Frères Estienne, 1759 ainsi qu'un «Mémoire sur l'instruction des paysans" dans le numéro de décembre 1771 du Journal de l'Agriculture, du Commerce, des Arts et des Finances (Paris, p. 22-45).

48. Eléments d'Agriculture, Paris, H. L. Guérin et L. F. Delatour, 1762, vol. I, p. X. Cité dans André Bourde, Agronomie et agronomes en France au XVIII', Paris, SEVPEN, 1967, vol. 2, p. 1057. 
Cette volonté de diffuser les méthodes agraires par le canal des ecclésiastiques sera reprise en France par bien d'autres. Et c'est dans cette optique que seront par exemple publiés bon nombre de catéchismes d'agriculture, petits manuels très simples, à vocation divulgatrice, dans lesquels préceptes moraux, religieux et agronomiques sont mêlés et exposés sous une forme populaire de dialogues ou de maximes que l'on retiendra facilement. C'est ainsi que l'Abbé Bexon, par exemple, publiera en 1773, à Paris, le Catéchisme d'Agriculture ou Bibliothèque des gens de la campagne dans laquelle on enseigne, par des procédés très simples, l'art de cultiver la terre,... et de rendre les hommes qui la cultivent, meilleurs et plus heureux. On retrouve également cette nécessité d'associer les ecclésiastiques au développement agraire des campagnes en Italie où Francisco Griselini publia en 1778 un ouvrage de quelque 90 pages au titre fort significatif : Discurso sobre el problema de si corresponde a los Párrocos y curas de las aldeas el instruir a los labradores en los buenos elementos de la Economía campestre; al cual va adjunto un plan que debe seguirse en la formación de una obra dirigida a la mencionada instrucción. Quelques années plus tard, en 1789, cette œuvre, qui ne devait pas passer inaperçue, fut traduite en espagnol par la célèbre Josefa Amar y Borbón, membre de la Real Sociedad Aragonesa de Amigos del País et ce, à la demande expresse de ladite institution. Pourquoi cette traduction ? Les sociétaires s'en expliquèrent longuement dans le Prologue. Pleinement conscients du retard considérable pris par l'Espagne dans le domaine des sciences, ils insistèrent tout particulièrement sur la nécessité impérieuse qu'il y avait à tirer profit des progrès réalisés en la matière par les nations étrangères. D'où l'importance accordée aux traductions :

Necesitamos - affirmaient-ils avec force - poner en nuestra lengua lo mucho que falta, y se halla en una serie de Escritores ilustres de otras Naciones, y principalmente en las Actas de la Regia Sociedad de Londres, en las de la Real Academia de París, en las famosas Sociedades de Bretaña, Berna, Dublín, y en las innumerables de otros Países, que formarían un inmenso Catalógo si las hubiésemos de referir ${ }^{49}$.

49. Francisco Griselini, Discurso sobre el problema de si corresponde a los Párrocos y Curas de las Aldeas el instruir a los labradores en los buenos elementos de la economía campestre : al cual va adjunto un plan que debe seguirse en la formación de una obra dirigida a la mencionada instrucción del Señor Francisco Griselini, Miembro de las principales Academias de Europa, y Secretario de la Sociedad Patriótica de Milán. Rusticationem creatam ab altissimo. Traducido del Italiano, por encargo de la Real Sociedad Aragonesa de Amigos del País, por Doña Josepha Amar y Borbón, Socia de mérito de la misma, Zaragoza, por Blas Miedes, Impresor de la Real Sociedad, 1789 , p. 4. 
C'est ainsi que la Société offrit des prix à ceux qui traduiraient divers ouvrages parmi lesquels les Eléments d'Agriculture de Duhamel dans lesquels l'accent fut mis, notamment, sur l'importance du rôle à jouer par les ecclésiastiques qui se devaient d'endosser l'habit d'instituteurs agricoles. Mettant en pratique ces bonnes paroles, la Real Sociedad Aragonesa... demanda à Josefa Amar y Borbón de traduire le fruit des travaux de Griselini qui étaient susceptibles de venir à bout de bien des idées reçues en démontrant aux ecclésiastiques espagnols que leur tâche ne pouvait se limiter au domaine du spirituel et qu'en se désintéressant des questions agraires ils entravaient considérablement le progrès de la plus importante des sciences :

Sin embargo, como uno de los principales impedimentos para su progreso es persuadirse muchas personas Eclesiásticas, que podían cooperar con sus luces y facultades al sistema benéfico de la Sociedad, que es ajeno de su sagrado Ministerio contribuir a esta causa común por otra vía que la puramente espiritual, pensó [la Société économique] arreglar un Discurso que demostrase, que todo buen ciudadano, y especialmente las personas distinguidas de su respetable Clero no estaban dispensadas de otras obligaciones, ni como Vasallos del Rey, ni como Ministros del Sacrosanto Altar ${ }^{50}$.

Autrement dit, il s'agissait de rappeler sans détour aux membres du clergé qu'ils n'étaient pas seulement des serviteurs de Dieu, mais qu'ils étaient aussi des citoyens et des sujets du Roi. Or, à ce titre, ils avaient pour obligation de s'associer à l'effort général et d'œuvrer, à leur tour, pour le bien de la nation, au développement de l'agriculture. Comment ? En s'adonnant à son étude et en faisant partager leur savoir au peuple. Il ne fait aucun doute que les membres de la vénérable institution partageaient en tous points les théories de Griselini lorsque celui-ci déclarait :

El arte de la Agricultura, siendo la más necesaria de todas [las artes], está por nuestra desgracia entregada al descuido de la casualidad, e ignorancia, no obstante que todas las clases del cuerpo político dependen de ella y de los que la practican; por lo que es absolutamente necesario que los Párrocos, y en particular los de las Aldeas la entiendan, así para cumplir por este medio una de sus principales obligaciones, según hemos demostrado, como para desempeñar la mayor y la más esclarecida obra de misericordia, cual es enseñar a sus semejantes que lo necesitan el medio de adquirir las comodidades de la vida, y ser útiles al mismo tiempo a otros, a la patria, a sus artes y en una palabra a la Repúblicam ${ }^{51}$.

50. Ibid., p. 5.

51. Ibid., p. 17-18. 
La Real Sociedad Vascongada de los Amigos del País, tout aussi attentive à la question, ne demeura pas en reste. Ainsi offrit-elle en 1790 un prix de 15 doublons et le titre de membre bienfaiteur à celui qui démontrerait que le clergé espagnol pouvait contribuer au bonheur physique et moral du peuple tout en stipulant les moyens d'y parvenir ${ }^{52}$. Le mémoire primé par les sociétaires, El Padre de su Pueblo o medios para hacer temporalmente felices a los pueblos con el auxilio de los señores Párrocos, portait la signature d'un éminent ecclésiastique, Pedro Díaz de Valdés, évêque et inquisiteur de Barcelone qui, pour l'occasion, adopta le pseudonyme de Zadivaldés. Cet ouvrage, qui sortit des presses en 1793 et ce à l'initiative de la Vascongada, bénéficia par ailleurs d'une publicité non négligeable. En effet, le Memorial literario, périodique renommé qui avait déjà ouvert ses pages à plusieurs écrits du même auteur, en fit en juin 1794 un enthousiaste et détaillé compte rendu où l'on pouvait lire en guise de conclusion :

Creemos que el libro de la Educación de los artesanos, y esta Memoria para la de los Eclesiásticos, harán época en la nación para beneficio de ambas clases; y ojalá que las otras hallen plumas que tracen la que convenga darlas. Si se ejecuta lo que propone el Autor de la Memoria, adelantará en instrucción provechosa, y lograrán todas las clases del estado, y más inmediatamente los pueblos cortos que son infinitos, un cúmulo de felicidades que apenas se sujeta al guarismo. Los extranjeros, leyendo esta Memoria, conocerán que no se ignoran en España las ciencias útiles; y que un Eclesiástico enseña el medio de hacerlas comunes, y generalmente provechosas a todas clases $^{53}$.

52. «Noticia de la Memoria sobre las felicidades físicas y morales que los Curas pueden procurar a los pueblos, y sobre los medios con que se proporcionarán para causar el bien común en todos ellos...” in El Padre de su Pueblo, o medios para hacer temporalmente felices a los pueblos, con el auxilio de lo Señores Curas párrocos. Memoria premiada por la Real Sociedad Vascongada e impresa de su orden en Victoria en 1793. Reimpresa ahora, con un discurso previo y algunas notas, con permiso de su autor, el Excmo Señor Don Pedro Díaz de Valdés, Caballero Gran Cruz de la Real distinguida orden española de Carlos tercero, del Consejo de S. M. y Obispo de Barcelona, Barcelona, Oficina de Manuel Tejero, 1806, p. 2-3 : «Es notorio que la Clerecía Española empleó su ciencia y dotación para remediar las miserias, y para aumentar los provechos de todos los ciudadanos. Pero aún se suspiraba por una obra que demostrara, que el Clero Español puede causar las felicidades físicas y morales de los pueblos; y quc indicara los medios, con que llegara efectivamente a producir tanto bien. Anunció esta dichosa idea la Sociedad Vascongada, ofreciendo en 1790 un premio de 15 doblones, y patente de Socio benemérito a quien desempeñase a su satisfacción tan interesante asunto.»

53. Memorial literario, Madrid, Imprenta Real, juin 1794. Texte reproduit dans l'introduction à l'édition de 1806 de El Padre de su Pueblo..., op. cit., p. 9-10. 
L'œuvre qui avait suscité l'admiration des rédacteurs du Memorial literario et recueilli par ailleurs l'assentiment du public fut même rééditée en 1806 , après que l'auteur ait procédé lui-même à un certain nombre d'additions. Et l'on retrouve bien évidemment dans le mémoire de celui qui percevait le clergé paroissial comme le canal le plus approprié pour contribuer au développement des campagnes et qui souhaitait que l'on offre aux curés un enseignement incluant des matières comme la botanique, la minéralogie et la chimie ${ }^{54}$, l'une des idées directrices du Semanario de Agricultura y Artes dirigido a los Párrocos qui n’a donc guère innové en voulant associer le clergé aux progrès de l'Agriculture. En revanche, le fait de vouloir associer presse et clergé, de mettre simultanément ces deux moyens de communication au service de l'agriculture, du commerce et des arts, constituait bel et bien une première. Malheureusement les résultats obtenus ne furent pas toujours à la hauteur des espérances de Godoy.

54. A ce sujet voir tout particulièrement la deuxième partie de son mémoire, intitulée "Medios con que los curas podrán ayudar a la felicidad, prosperidad y riqueza de los pueblos", p. 85-172. 